RAIRO Operations Research

RAIRO Oper. Res. 39 (2005) 3-12

DOI: $10.1051 /$ ro:2005004

\title{
A MATHEMATICAL MODEL FOR AN OPTIMAL APPOINTED DELIVERY DATE ON A HOME DELIVERY MARKET
}

\author{
Hidefumi KaWAKatsu ${ }^{1}$ AND Hiroaki Sandoh ${ }^{2}$
}

\begin{abstract}
In recent years, the home delivery market has rapidly been growing since customers can purchase a variety of products very easily via Internet. At the same time, however, customers tend to switch from a supplier to another seeking for better service for them. For this reason, it is necessary for suppliers to enclose their customers by means of various kinds of service and strategy. An appointed delivery date of a product ordered by a customer is one of important factors of supplier's services. From the suppliers' point of view, they hope to make the period from the order date to the delivery date as short as possible to increase their customers, but at the same time they prefer to make this period as long as possible since the risk becomes higher that they cannot deliver products to their consumer by the appointed date under the short period appointed date. This study proposes a stochastic model to determine an optimal appointed delivery date for a supplier. For small values of an appointed delivery date $L$, the probability that a customer purchases the product becomes larger, but the probability of tardiness increases. In contrast, the purchase probability as well as the penalty of tardiness decreases with $L$. From this point of view, this study formulates the expected profit for a supplier, which is to be maximized as an objective function. Clarified are the conditions under which an optimal appointed delivery date exists for the case where the purchase probability is expressed by a multinomial logit model. Numerical examples are also presented.
\end{abstract}

Keywords. Home delivery service, expected profit per customer, optimal appointed delivery date, multinomial logit model.

Received April, 2004. Accepted November, 2005.

${ }^{1}$ Department of Information \& Economics, University of Marketing and Distribution Sciences, Nishi, Kobe 651-2188 Japan; Hidefumi_Kawakatsu@red.umds.ac.jp

2 Department of Business Administration, Kobe Gakuin University, Nishi, Kobe 651-2188 Japan; sandoh@ba.kobegakuin.ac.jp 


\section{INTRODUCTION}

Home delivery service represents a service that suppliers offer to bring or transport products to convenient places for customers. Home delivery market has been growing rapidly since customers can purchase a variety of products very easily via Internet. At the same time, however, customers tend to switch from a supplier to another seeking for better service for them[1]. For this reason, it is necessary for suppliers to enclose their customers by means of various kinds of service and strategy.

An appointed delivery date of a product ordered by a customer is one of important factors of supplier's services. From the suppliers' point of view, they hope to make the period from the order date to the delivery date as short as possible to increase their customers, but at the same time they prefer to make this period as long as possible since the risk becomes higher that they cannot deliver products to their customers by the appointed date under the short period appointed date.

Several models to determine an optimal date of delivery have been proposed [2-14]. Çetinkaya and Lee [2] and Chen and Samroengraja [3] have determined an optimal dispatch time of products under the circumstances where, if the supplier postpones the dispatch time, the transportation cost decreases and therefore the supplier can dispatch a large quantity, but the cost for keeping the customer waiting becomes large.

$\mathrm{Li}$ and Cheng[4] have considered a due-date determination problem, which attempts to minimize the tardiness penalty plus due-date assignment cost. They showed the conditions where their problem is solvable in polynomial time. Dewan and Mendelson[5] carried out the trade-offs between the delay cost and the capacity cost. They were justified in concentrating on the properties of the solutions to the first-order conditions. Stidham[6] has dealt with a model, which can be viewed as a variant of the model by Dewan and Mendelson[5]. They confined themselves into quantitative analysis of the model in order to complement to Dewan and Mendelson's analysis of its qualitative properties. They introduced an upper bound on the arrival rate which is a parameter of the price, and examined the existence of multiple solutions to the first-order conditions. They also developed iterative algorithms that converge to a local optimum or a global one. Palaka et al.[7], So and Song $[8]$ and Boyaci and Ray $[9]$ have introduced a probability constraint to ensure that a satisfactory service level is achieved when the delivery time guarantee is selected. Palaka et al.[7] and So and Song[8] have treated the waiting time in an $M / M / 1$ queue to make the problem more tractable since it is well known that the waiting time is exponentially distributed for an $M / M / 1$. They developed a simple procedure to obtain a solution from which they characterized the optimal pricing, delivery time guarantees and capacity expansion decisions. So and Song [8] also showed that the tail of the waiting time distribution for high service levels is well approximated by an exponential distribution even for a $G / G / s$ queue. Boyaci and Ray[9] have considered a profit-maximizing firm that sells two substitutable products in a price and time sensitive market for high service level. They assumed that a firm dealt in both regular (slower) products and express (faster) ones. 
They discussed a method for determining an optimal delivery time of the express product and appropriate prices of the two products, taking into account the impact of delivery time reduction on capacity requirements and costs.

Several studies have also been conducted from game theory approach to determine an optimal price and delivery-time [10-14]. Kalai et al.[10] have studied competition in service rate, and $\operatorname{Li}[11]$ has considered competitive choice of inventory level. In both articles, however, it is assumed that customers make their decisions based on only the speed of delivery because the prices and quality levels are fixed and identical. Li and Lee[12] have discussed the price competition between two service firms, where customers consider both their prices and delivery speeds in selecting their service. Lederer and $\mathrm{Li}[13]$ have developed an analytical model that captures the effect of time performance on prices, demands and profit. They considered the situation where firms compete by choosing prices and service rates for multiple types of customers with different delay costs. Their results show how the different firms would choose their price and time performance for these different customer types. So[14] has extended the single firm model developed by So and Song [8] to a $N$-firm problem. They showed that a unique Nash equilibrium exists and presented an iterative procedure to obtain the equilibrium.

However, there still remain many problems unsolved in determining an optimal appointed delivery date under the circumstances where each customer chooses the single option that yields the greatest utility, while the utility is described as a random variable reflecting unobservable taste differences. Under these circumstances, the probability that a customer chooses the a firm can be given by the discrete choice model[15]. The discrete choice models have seen extensive applications in the fields of economics, transportation, and marketing among many others [15-19].

This study proposes a stochastic model to determine an optimal appointed delivery date for a supplier. The expected profit for a supplier is formulated as an objective function to be maximized. Clarified are the conditions under which an optimal appointed delivery date exists in the case where the purchase probability is given by a multinomial logit model. Numerical examples are also presented to illustrate the theoretical underpinnings of the proposed formulation.

\section{MODEL}

The main notations used in this paper are listed below:

$L$ : Appointed delivery date of the product ordered by a customer (decision variable).

$q(L)$ : Probability of a customer's purchasing the product $(q(L) \geq 0)$.

$X:$ Lead time from the order date to the delivery date. This is called delivery lead time.

$T: \quad$ Tardiness $(=T=\max (0, X-L))$.

$c$ : Penalty of tardiness per customer per unit of tardiness.

a: $\quad$ Gross profit per customer. 
The assumptions in this study are as follows:

(i) Purchase probability $q(L)$ is non-increasing in delivery date $L$.

(ii) Delivery lead time $X$ is a random variable which follows a general distribution with the cumulative distribution function $(c d f) F(\cdot)$ and the probability density function $(p d f) f(\cdot)$. We assume $E[X]=\mu \ll+\infty$.

The penalty $P(L, X)$ for tardiness per customer is given by

$$
P(L, X)=\max [0, c(X-L)],
$$

and then the expected penalty for tardiness per customer becomes

$$
E[P(L, X)]=c \int_{L}^{+\infty}(x-L) f(x) \mathrm{d} x .
$$

Hence, the expected profit per single customer can be expressed by

$$
Q(L)=q(L)\left[a-c \int_{L}^{+\infty}(x-L) f(x) \mathrm{d} x\right] .
$$

It is easily proven that $Q(L)$ in equation (3) is strictly increasing and decreasing respectively in $a$ and $c$.

\section{Optimal appointed Delivery Date}

In this section, we focus on the case where a customer chooses the supplier which yields the largest utility for him: the purchase probability $q(L)$ in such a situation can be given by the discrete choice model [15] discussed in detail below.

Utility $U_{j}(j=1,2, \cdots, n)$ of a product from supplier $j$ can be decomposed as follows:

$$
U_{j}=V_{j}+\varepsilon_{j},
$$

where $V_{j}$ is called the observable or measured utility and reflects the preference of the customer, while $\varepsilon_{j}$ is a random variable which varies with their idiosyncratic taste differences.

In most models using the discrete choice model, $V_{j}$ is given by

$$
V_{j}=\boldsymbol{\alpha}_{j}+\boldsymbol{\nu}^{T} \boldsymbol{z}_{j},
$$

where $\alpha_{j}$ is invariant preference for the product of supplier $j$, and $\boldsymbol{z}_{j}$ is a vector that includes marketing and other variables associated with the product of supplier $j$ [15-17]. Vectors $\boldsymbol{\nu}$ and $\boldsymbol{z}$ are respectively defined by $\boldsymbol{\nu}^{T}=\left(\nu_{1}, \nu_{2}, \cdots, \nu_{s-1}\right.$, $\left.\lambda, \nu_{s+1}, \cdots, \nu_{m}\right)$ and $\boldsymbol{z}=\left(\boldsymbol{z}_{1}^{T}, \boldsymbol{z}_{2}^{T}, \cdots, \boldsymbol{z}_{n}^{T}\right)$ with $\boldsymbol{z}_{j}=\left(z_{1 j}, z_{2 j}, \cdots, z_{m j}\right)(j=$ $1,2, \cdots, n, j \neq J)$ and $\boldsymbol{z}_{J}=\left(z_{1 J}, z_{2 J}, \cdots, z_{(s-1) J},-L, z_{(s+1) J}, \cdots, z_{m J}\right)$. 
The $s$ th component $\lambda$ of $\boldsymbol{\nu}$ represents a common parameter to all the suppliers, which is associated with the delivery date.

If random variable $\varepsilon_{j}$ in equation (4) independently and identically follows a double exponential distribution, then the probability $q(L)$ that the objective customer purchases the product from supplier $J$ is, in a closed form, given by

$$
\begin{aligned}
q(L) & =\frac{\mathrm{e}^{\alpha_{J}+\boldsymbol{\nu}^{T} \boldsymbol{z}_{J}}}{\sum_{j=1}^{n} \mathrm{e}^{\alpha_{i}+\boldsymbol{\nu}^{T} \boldsymbol{z}_{i}}} \\
& =\frac{\mathrm{e}^{\alpha_{J}+\sum_{i \neq s} \nu_{i} z_{i J}-\lambda L}}{\sum_{j \neq J} \mathrm{e}^{\alpha_{j}+\boldsymbol{\nu}^{T} \boldsymbol{z}_{j}}+\mathrm{e}^{\alpha_{J}+\sum_{i \neq s} \nu_{i} z_{i J}-\lambda L}},
\end{aligned}
$$

which is well known as the discrete choice multinomial logit model [15-19].

Let $B_{1}$ and $B_{2}$ respectively be given by

$$
\begin{aligned}
& B_{1}=\sum_{j \neq J} \mathrm{e}^{\alpha_{j}+\boldsymbol{\nu}^{T} \boldsymbol{z}_{j}}, \\
& B_{2}=\alpha_{J}+\sum_{i \neq s} \nu_{i} z_{i J}
\end{aligned}
$$

then $q(L)$ in equation (6) can be rearranged as

$$
q(L)=\frac{\mathrm{e}^{B_{2}-\lambda L}}{B_{1}+\mathrm{e}^{B_{2}-\lambda L}},
$$

and consequently, the expected profit per customer for supplier $J$ is expressed by

$$
Q(L)=\frac{\mathrm{e}^{B_{2}-\lambda L}}{B_{1}+\mathrm{e}^{B_{2}-\lambda L}}\left[a-c \int_{L}^{+\infty}(x-L) f(x) \mathrm{d} x\right]
$$

By differentiating $Q(L)$ in equation (10) with respect to $L$, we have

$$
\begin{aligned}
Q^{\prime}(L)= & -\frac{\mathrm{e}^{B_{2}-\lambda L}}{B_{1}+\mathrm{e}^{B_{2}-\lambda L}} \\
& \times\left\{\frac{B_{1} \lambda}{B_{1}+\mathrm{e}^{B_{2}-\lambda L}}\left[a-c \int_{L}^{+\infty}(x-L) f(x) \mathrm{d} x\right]-c \bar{F}(L)\right\} .
\end{aligned}
$$

Then $Q^{\prime}(L) \geq 0$ agrees with

$$
\int_{L}^{+\infty}(x-L) f(x) \mathrm{d} x+\frac{B_{1}+\mathrm{e}^{B_{2}-\lambda L}}{B_{1} \lambda} \bar{F}(L) \geq \frac{a}{c} .
$$


Let $A(L)$ express the left-hand-side of Inequality (12), we have

$$
\begin{aligned}
A^{\prime}(L) & =-\bar{F}(L)-[\lambda \bar{F}(L)+f(L)] \frac{B_{1}+\mathrm{e}^{B_{2}-\lambda L}}{B_{1} \lambda}(<0), \\
\lim _{L \rightarrow+0} A(L) & =\mu+\frac{B_{1}+\mathrm{e}^{B_{2}}}{B_{1} \lambda} \\
\lim _{L \rightarrow+\infty} A(L) & =0\left(<\frac{a}{c}\right) .
\end{aligned}
$$

These observations can clarify the conditions where an optimal appointed delivery date $L^{*}$ exists.

(1) $\mu+\frac{B_{1}+\mathrm{e}^{B_{2}}}{B_{1} \lambda}>\frac{a}{c}$ : in this subcase, the sign of $Q^{\prime}(L)$ varies from positive to negative only once, and thus there exists a unique finite positive $L^{*}$. The expected profit per customer becomes

$$
Q\left(L^{*}\right)=\frac{c \mathrm{e}^{B_{2}-\lambda L^{*}}}{B_{1} \lambda} \bar{F}\left(L^{*}\right) .
$$

(2) $\mu+\frac{B_{1}+\mathrm{e}^{B_{2}}}{B_{1} \lambda} \leq \frac{a}{c}$ : in this subcase, we have $Q^{\prime}(L) \leq 0$ and therefore $L^{*} \rightarrow 0$. The expected profit per customer is given by

$$
Q\left(L^{*}\right)=\frac{\mathrm{e}^{B_{2}}}{B_{1}+\mathrm{e}^{B_{2}}}(a-c \mu) .
$$

\section{Numerical EXAMPLES}

This section presents numerical examples to illustrate the proposed model under the circumstances where the probability $q(L)$ is given by the discrete choice multinomial logit model.

Suppose that the lead time, $X$, follows a two parameter exponential distribution with a density function

$$
f(x)=\left\{\begin{array}{cc}
0, & \text { if } x<\theta, \\
\gamma \mathrm{e}^{-\gamma(x-\theta)}, & \text { if } x \geq \theta,
\end{array}\right.
$$

and then $Q(L)$ in equation (3) can be rewritten as

$$
Q(L)=\frac{\mathrm{e}^{B_{2}-\lambda L}}{B_{1}+\mathrm{e}^{B_{2}-\lambda L}}\left[a-\frac{c}{\gamma} \mathrm{e}^{-\gamma(L-\theta)}\right] .
$$


It is easily proven that $Q(L)$ in equation (19) is strictly increasing and decreasing in $\gamma$ and $\theta$, respectively.

We consider the case where there exists three suppliers, and we confine ourselves into supplier $J=1$. Let $\boldsymbol{\alpha}, \boldsymbol{\nu}$ and $\boldsymbol{z}$ be, respectively, given by

$$
\begin{aligned}
\boldsymbol{\alpha} & =(1.0,1.0,1.0), \\
\boldsymbol{\nu}^{T} & =(\lambda, 0.3,0.3),
\end{aligned}
$$

and

$$
\boldsymbol{z}=\left(\begin{array}{ccc}
-L & -1.0 & 1.0 \\
-3.0 & -1.2 & 1.2 \\
-2.0 & -1.5 & 1.4
\end{array}\right)
$$

Further, let $(\theta, a)=(1.0,100.0)$, and then we have

$$
q(L)=\frac{\mathrm{e}^{B_{2}-\lambda L}}{B_{1}+\mathrm{e}^{B_{2}-\lambda L}}
$$

where we have, from equations (7) and (8),

$$
\begin{aligned}
& B_{1}=\mathrm{e}^{1-3 \lambda}+\mathrm{e}^{0.97-2 \lambda}, \\
& B_{2}=1.0 .
\end{aligned}
$$

Figure 1a shows the behavior of $Q(L)$ when $L$ varies in the case of $(\lambda, \gamma)=(1.0,1.0)$ with $c=200,250$ and 300. Figure 1a indicates that $Q(L)$ is unimodal in relation to $L$, and this figure corresponds to subcase (1) in Section 3. It is also observed in Figure 1a that $Q(L)$ becomes smaller when $c$ becomes large, which can be understood from equation (19).

Figure 1b depicts the curves of $Q(L)$ in the case of $(\gamma, c)=(1.0,200.0)$ with $\lambda=0.5,1.0$ and 3.0. It is observed in this figure that the shape of $Q(L)$ is remarkably affected by $\lambda$, but this is due to the behavior of $q(L)$, which is revealed in Figure 2.

Figure 1c illustrates the behavior of $L^{*}$ along with that of $Q\left(L^{*}\right)$ when $\lambda$ changes in the case of $\gamma=1.0$ with $c=200.0,250.0$ and 300.0. Figure 1 c reveals that $L^{*}$ decreases on the whole when $\lambda$ becomes larger. This is because when $\lambda$ takes a large value, the appointed delivery date becomes a relatively important factor that stimulates the customer to purchase a product from the supplier. It is also seen in Figure 1c that $Q\left(L^{*}\right)$ is decreasing in $\lambda$ and $c$.

Figure $1 \mathrm{~d}$ shows the curves of $L^{*}$ and $Q\left(L^{*}\right)$ when $\lambda$ varies in the case of $c=200.0$ with $\gamma=0.3,0.6$ and 1.0. In Figure $1 \mathrm{~d}$, we can observe that both $L^{*}$ and $Q\left(L^{*}\right)$ decreases with increasing $\lambda$, which was also observed in Figure 1c. It is also seen in Figure 1d that $L^{*}$ tends to decrease when $\gamma$ becomes larger. Since $\gamma$ denotes the scale parameter of the underlying distribution to express the random behavior of actual lead time, the variance of actual lead time increases with $\gamma$, 


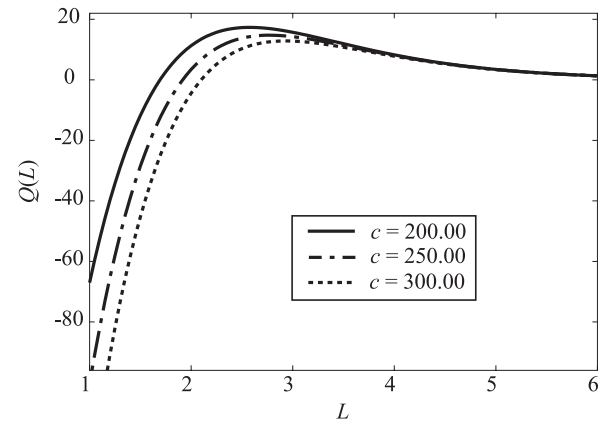

(a) Behavior of $Q(L)$

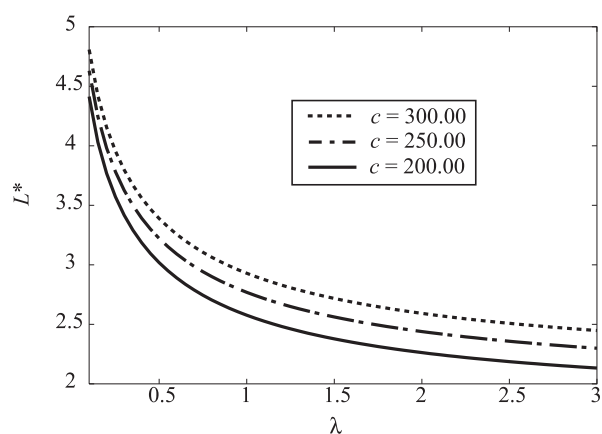

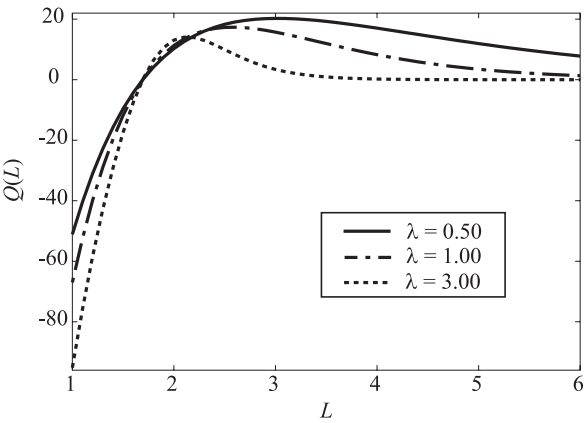

(b) Behavior of $Q(L)$

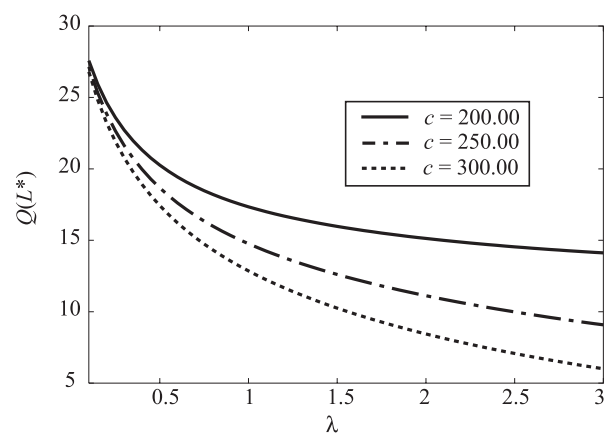

(c) Sensitivity analysis with respect to $\lambda$ and $c$
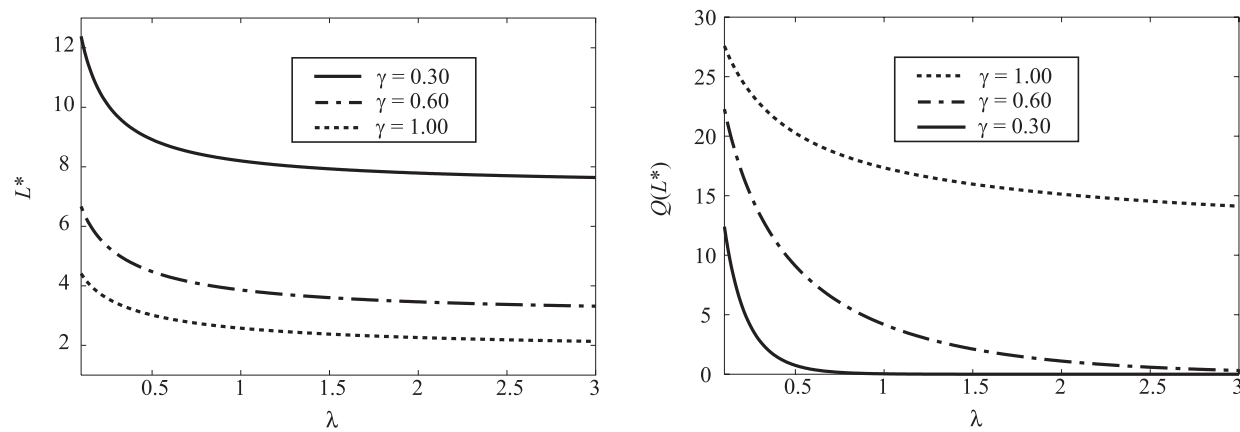

(d) Sensitivity analysis with respect to $\lambda$ and $\gamma$

FIGURE 1. Sensitivity analysis.

that is, the uncertainty becomes large whether or not the supplier can deliver the ordered product to the consumer by the appointed delivery date. Hence, $L^{*}$ and $Q\left(L^{*}\right)$ become small as $\gamma$ increases. 


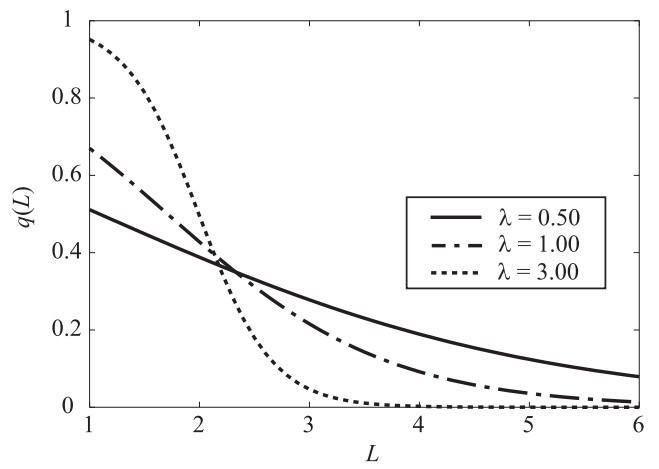

Figure 2. Behavior of $q(L)$.

\section{CONCLUSIONS}

This paper proposed a stochastic model to determine an optimal appointed delivery date for a supplier. The expected profit for a supplier was formulated which was to be maximized as an objective function. We focused on the case where the purchase probability $q(L)$ with the appointed delivery date $L$ was given by the multinomial logit model, and clarified the conditions under which an optimal appointed delivery date existed. Numerical examples were the presented to illustrate the proposed model.

We can easily extend our model so that it can express the situation where the supplier can receive some additional profit if he delivers the ordered product to the customer before due date. In addition, it is well-known that the multinomial logit model is based on and restricted by IIA (Independence from Irrelevant Alternatives) property. The relaxation of such a restriction is also an interesting extension.

\section{REFERENCES}

[1] J. Gattorna, Strategic supply chain alignment: best practice in supply chain management. Gower Publishing Limited, Hampshire England (1998).

[2] S. Çetinkaya and C. Lee, Stock replenishment and shipment scheduling for vendor-managed inventory systems. Manage. Sci. 46 (2000) 217-232.

[3] F. Chen and R. Samroengraja, A staggered ordering policy for one-warehouse, multiretailer systems. Oper. Res. 48 (2000) 281-293.

[4] C. Li and T.C.E. Cheng, due-date determination with resequencing. IIE Transactions 31 (1999) $183-188$

[5] S. Dewan and H. Mendelson, User delay costs and internal pricing for a service facility. Manage. Sci. 36 (1990) 1502-1517.

[6] S. Stidham, JR., Pricing and capacity decisions for a service facility: Stability and multiple local optima. Manage. Sci. 38 (1992) 1121-1139.

[7] K. Palaka, S. Erlebacher and D.H. Kropp, Lead-time setting, capacity utilization, and pricing decisions under lead-time dependent demand. IIE Transactions 30 (1998) 151-163. 
[8] K.C. So and J. Song, Price, delivery time guarantees and capacity selection. Eur. J. Oper. Res. 111 (1998) 28-49.

[9] T. Boyaci and S. Ray, Product differentiation and capacity cost interaction in time and price sensitive markets. Manufact. Service Oper. Manage. 5 (2003) 13-36.

[10] E. Kalai, M.I. Kamien and M. Rubinovitch, Optimal service speeds in a competitive environment. Manage. Sci. 38 (1992) 1154-1163.

[11] L. Li, The role of inventory in delivery-time competition. Manage. Sci. 38 (1992) 182-197.

[12] L. Li and Y.S. Lee, Pricing and delivery-time performance in a competitive environment. Manage. Sci. 40 (1994) 633-646.

[13] P.J. Lederer and L. Li, Pricing, production, scheduling, and delivery-time competition. Oper. Res. 45 (1997) 407-420.

[14] K.C. So, Prince and time competition for service delivery. Manufact. Service Oper. Manage. 2 (2000) 392-409.

[15] S.P. Anderson, Discrete choice theory of product differentiation. MIT Press, Cambridge (1992).

[16] S. Basuroy and D. Nguyen, Multinomial logit market share models: equilibrium characteristics and strategic implications. Manage. Sci. 44 (1998) 1396-1408.

[17] P.K. Chintagunta, Measuring the effects of new brand introduction on inter-brand strategic interaction. Eur. J. Oper. Res. 118 (1999) 315-331.

[18] W. Hanson and K. Martin, Optimizing multinomial logit profit functions. Manage. Sci. 42 (1996) 992-1003.

[19] J.J. Louviere, D.A. Hensher and J.D. Swait, Stated choice methods. Cambridge University Press (2000) 